though most of their data are described fairly well by this behavior. The most notable deviation is the forward angle point $\left(28^{\circ} \mathrm{cm}\right.$. $)$ at $980 \mathrm{Mev}$, for which the value of $[p(1000) / p(k)] \sigma(\theta)$ is $(0.84 \pm 0.11) \times 10^{-31} \mathrm{~cm}^{2} / \mathrm{sr}$ compared to an average value of 1.25 or $1.3 \times 10^{-31}$ $\mathrm{cm}^{2} / \mathrm{sr}$ for their data at $1010 \mathrm{Mev}$, and 1.02 to 1.15 $\times 10^{-31} \mathrm{~cm}^{2} / \mathrm{sr}$ for the other angles at $980 \mathrm{Mev}$. This rapid decrease in the forward angle cross section toward low photon energies was not observed in our experiment.

The difference of roughly $25 \%$ between the Cornell average for $[p(1000) / p(k)] \sigma(\theta)$ and ours is presumably a measure of the difference in the systematic errors of the two experiments.

A comparison of the experimental data with the available theories was discussed by McDaniel et al., $0^{4,5}$ and we refer their article for this discussion.

\section{ACKNOWLEDGMENTS}

We would like to thank $R$. Diebold for help in analyzing the data, E. Emery for maintenance of the hydrogen target, $\mathrm{J}$. Boyden for aid in constructing the fan counters, and A. Neubeiser and other members of the laboratory for assistance in the operation of the synchrotron. One of us, A.M.W., would like to thank Professor R. F. Bacher and the members of the Synchrotron Laboratory for their kind hospitality during his stay in CalTech. He is also grateful to the Commonwealth Fund of New York for a grant.

\title{
Experiment on Charge Independence in Interactions of Nucleons and Pions
}

\author{
D. Harting, J. C. Kuuyver, A. Kusumegi,* R. Rigopoulos, $\dagger$ A. M. Sachs, $\ddagger$ \\ G. Tibell, G. Vanderhaeghe, and G. Weber \\ CERN, Geneva, Switzerland
}

(Received April 18, 1960)

\begin{abstract}
The ratio $(R)$ of the differential cross sections of the reactions $p+d \rightarrow \mathrm{H}^{3}+\pi^{+}$and $p+d \rightarrow \mathrm{He}^{3}+\pi^{0}$ has been measured at a bombarding energy $E_{p}=591 \mathrm{Mev}$. The $\mathrm{He}^{3}$ and $\mathrm{H}^{3}$ nuclei are detected by the combination of magnetic deflection, time-of-flight measurement and pulse-height selection. The results are $R=2.26$ $\pm 0.11,2.10 \pm 0.14$, and $2.05 \pm 0.09$ at c.m. angles of the heavy particles of $52^{\circ}, 67^{\circ}$, and $143^{\circ}$, respectively. The agreement with the predictions of charge independence is satisfactory when the Coulomb and mass corrections are taken into account.
\end{abstract}

\section{INTRODUCTION}

$S^{\mathrm{I}}$ $\mathrm{INCE}$ the hypothesis of charge independence of nuclear forces was first formulated, this principle has, by its simplicity and general usefulness, contributed decisively to the understanding of a great variety of phenomena in nuclear physics. The fundamental nature of this principle and the associated concept of isotopic spin was strongly confirmed by the success of its application to pion-nucleon interactions.

The principle distinguishes between pure nuclear interactions which are assumed to be charge independent and other interactions, dependent on the charge of the elementary particles, that are responsible for Coulomb forces and mass effects. Every conclusion derived from charge-independent theory has therefore to be amended for the effect of these charge-dependent interactions, before a comparison with experimental data can be made. Whereas it is often possible to make predictions on the basis of charge independence, which are independent of the model assumed in the theoretical treatment of nuclear structure, such a specific model is in general required for the calculation of the Coulomb

\footnotetext{
* Now at University of Kyoto, Kyoto, Japan.

$\dagger$ Now at University of Birmingham, Birmingham, England.

$\ddagger$ National Science Foundation Senior Postdoctoral Fellow on leave from Columbia University 1958-1959, New York, New York.
}

corrections. The most critical tests are thus provided by those experiments for which the Coulomb corrections can be calculated and be shown to be small. The number of pertinent experiments in pion physics which can be performed with high accuracy is rather limited. Many of the proposed experimental tests involve the detection of neutral particles for which the techniques are not yet sufficiently developed to reach high experimental precision. It was recognized early that this difficulty can be avoided in the experiment which is the subject of the present paper. According to charge independence the angular distribution of the reactions:

and

$$
\begin{aligned}
& p+d \rightarrow \mathrm{H}^{3}+\pi^{+}, \\
& p+d \rightarrow \mathrm{He}^{3}+\pi^{0}
\end{aligned}
$$

are the same and the ratio of the absolute cross sections $(R)$ is $2 .^{1}$ As the cross sections of these reactions may be obtained by detecting only the $\mathrm{H}^{3}$ and $\mathrm{He}^{3}$ nuclei, which are produced by the same proton beam for both reactions, no neutral particles are involved in the measurement. Experimentally the main problem is the small cross section of these processes compared to those 1 A. M. L. Messiah, Phys. Rev. 86, 430 (1952); M. Ruderman,
Phys. Rev. 87, 383 (1952). 
of competing reactions. Once this difficulty is overcome by the use of a very selective mass-three particle detector and a high-intensity proton beam, the experiment can be performed with an accuracy which is typical for all experiments involving charged particles only, i.e., 3-5\%.

The first studies of these reactions with a lowintensity $340-\mathrm{Mev}$ proton beam were not sufficiently accurate to be considered as a strict test of charge independence, but the occurrence of both reactions was unequivocally established and the angular distribution of reaction (1) was measured. ${ }^{2}$

In the present experiment with the 591-Mev protons of the CERN synchrocyclotron a final precision of $3 \%$ is obtained, which is equal to the uncertainty in the calculation of the Coulomb and mass corrections. In a similar experiment in Chicago $^{3}$ a precision of $7 \%$ was reached at $E_{p}=450 \mathrm{Mev}$. In this case the center-ofmass energy of the outgoing pions is $140 \mathrm{Mev}$, which is below the $\left(\frac{3}{2}, \frac{3}{2}\right)$ resonance energy. The $591-\mathrm{Mev}$ protons produce in these reactions pions of $220-\mathrm{Mev}$ c.m. energy, which is on the downward slope of this resonance. In this respect, the two experiments provide mutually supplementary information on the validity of charge independence.

In this context it should be mentioned that a recent comparison $^{4}$ of the reactions $p+p \rightarrow d+\pi^{+}$and $n+p$ $\rightarrow d+\pi^{0}$ at $600 \mathrm{Mev}$ shows consistency with charge independence within the experimental errors, which are $10 \%$ for the angular distributions and $20 \%$ for the cross sections. Further information on experiments bearing on the validity of charge independence may be found in a review paper presented by Pontecorvo at the Kiev conference. ${ }^{5}$

The results which are presented in this paper were obtained in three cyclotron runs of about one week each. In the first run, as has already been reported briefly, ${ }^{6}$ the ratio $(R)$ of the differential cross sections of reactions (1) and (2) was measured at a c.m. angle of the heavy particle of $52^{\circ}$. In the second run $R$ was measured at a c.m. angle of $67^{\circ}$. In addition, a measurement was made of process (1) with the incident proton energy degraded by $10 \mathrm{Mev}$. From this measurement, a correction can be made for the difference in c.m. momenta of pions in (1) and (2) at the same proton

${ }^{2}$ W. J. Frank, K. C. Bandtel, R. Madey, and B. J. Moyer, Phys. Rev. 94, 1716 (1954); K. C. Bandtel, W. J. Frank, and

B. J. Moyer, Phys. Rev. 106, 802 (1957)

${ }^{3}$ A. V. Crewe, E. Garwin, B. Ledley, E. Lillethun, R. March, and S. Marcowitz, Phys. Rev. Letters 2, 269 (1959); A. V. Crewe, B. Ledley, E. Lillethun, S. Marcowitz, and C. Rey, Phys. Rev. 118,1091 (1960).

${ }^{4}$ V. B. Fliagin, V. P. Dzhelepov, V. S. Kiselev, and K. O. Oganesian, J. Exptl. Theoret. Phys. (U.S.S.R.) 35, 854 (1958) [translation: Soviet Phys. 35(8), 592 (1959)].

${ }^{5}$ B. Pontecorvo, Report to 1959 International Conference on High-Energy Physics, Kiev (unpublished).

${ }^{6} \mathrm{D}$. Harting, J. C. Kluyver, A. Kusumegi, R. Rigopoulos, A. M. Sachs, G. Tibell, G. Vanderhaeghe, and G. Weber, Phys Rev. Letters 3, 52 (1959). energy (resulting from the $\pi^{+}, \pi^{0}$ mass difference), in order to determine $R$ for emerging pions of the same c.m. momentum. In the third run $R$ was measured at a c.m. angle of $143^{\circ}$.

\section{EXPERIMENTAL METHOD}

\section{(A) General}

As mentioned in the introduction, the differential cross section of reactions (1) and (2) is measured by detecting only the outgoing $\mathrm{H}^{3}$ and $\mathrm{He}^{3}$ particles. This is possible as these reactions are two-body processes, and therefore the particles produced at a given angle have a well defined energy. The overwhelming number of other reaction products (protons, deuterons, and pions) discourages the detection of the pions in coincidence with the heavy particle as additional evidence for the correct identification of reactions (1) and (2).

To distinguish the $\mathrm{H}^{3}$ (or $\mathrm{He}^{3}$ ) particles from $p, d$, and $\pi$ 's, one has to submit the reaction products to some kind of mass analysis. In the present experiment this mass analysis is based on momentum selection through deflection in a magnetic field and velocity selection by measurement of the time of flight between two counters. The magnetic separation reduces the number of particles traversing the counter telescope and helps to avoid overloading of the coincidence system. Although, in principle, these two selection criteria are sufficient, additional information on the selected particles is derived from pulse-height analysis of the pulses in one of the counters. In runs 2 and 3 , in addition to the electronic analysis, photographs were taken of oscilloscope traces, which displayed the pulses from presumed mass-three particles in two counters. The differential cross sections of the two reactions are measured one after the other with the help of a proton beam monitor.

These general considerations lead to a layout of the experiment as shown in Fig. 1. The external proton beam bombards a liquid deuterium target and the emerging particles, that pass through the collimator and have the correct ratio of momentum to charge, are bent through an angle of $26.6^{\circ}$ by the analyzing magnet. The particles are then detected in the threecounter telescope, and those with a time-of-flight between counters 1 and 2 (and 3) corresponding to nuclei of mass three are recorded as possible events.

Deviations from the ideal situation of truly monoenergetic mass-three particles are introduced by energy loss in the target and the spread in the value of the production angle in the actual experiment. This spread is caused by divergence of the proton beam, the size of the proton beam spot and the dimensions and position of the collimator $(K 2)$. The distance between counters 1 and 2 was chosen to achieve complete timeof-flight separation between deuterons and tritons of the same momentum and between protons, $\mathrm{He}^{3}$ par- 


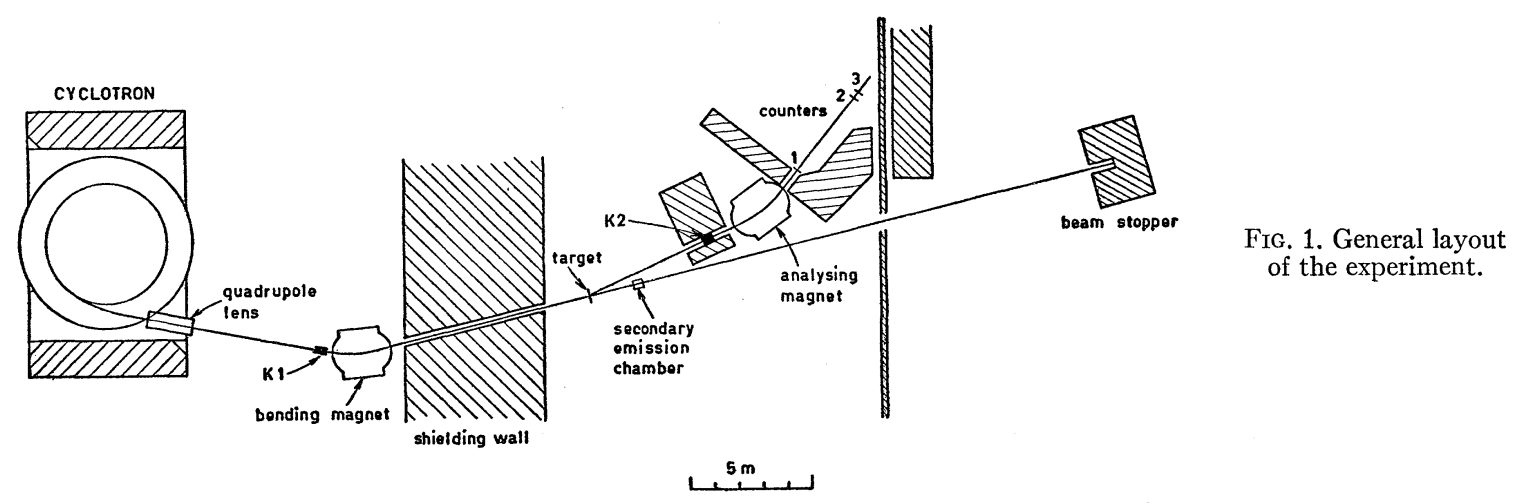

ticles, and deuterons of the same momentum over $Z$. The counters were made slightly larger than the calculated cross section of the beam (at the position of each of the counters) of $\mathrm{He}^{3}$ particles that pass through the collimator. Multiple scattering was kept to a minimum by using a thin first counter and hydrogen bags along the beam path. The parameters used in the experiment are listed in Table I.

The $\mathrm{H}^{3}$ and $\mathrm{He}^{3}$ particles have a c.m. velocity which is smaller than the velocity of the center-of-mass system itself. These particles appear therefore in the laboratory within a cone in the forward direction. At any angle in this cone, two groups of different energy are observed corresponding to $\mathrm{H}^{3}$ and $\mathrm{He}^{3}$ produced either in the forward or backward direction in the center-of-mass system. As the properties of the particles in the two groups differ considerably (see Table II), a check on systematic errors in the experiment is obtained by measuring the ratio for each of the two groups.

\section{(B) Proton Beam}

The proton beam is extracted by the regenerative method from the CERN synchrocyclotron. After leaving the cyclotron field, it passes in evacuated pipes through two quadrupole lenses and a wedge-shaped magnet, which deflects it over $26^{\circ}$ into a channel in the shielding wall. The combined effect of this magnet and the lenses is to produce a beam spot at the target position of approximately $2 \times 3 \mathrm{~cm}^{2}$. The angle of divergence is less than $\frac{1}{4}^{\circ}$.

From the extraction conditions the mean energy of the external proton beam is calculated to be $591 \pm 3$ Mev. The energy spread is less than $\pm 3 \mathrm{Mev}$.

The lower energy beam needed for the measurement of the energy dependence of the cross section was obtained by placing a polythene absorber of $3.6 \mathrm{~g} / \mathrm{cm}^{2}$ just before the quadrupole lenses. A second pair of quadrupole lenses was installed before the bending magnet to obtain a beam spot of $2 \times 3 \mathrm{~cm}^{2}$ similar to the full energy beam spot, without losing more than half of the beam intensity.

The proton beam is monitored by a secondary emission chamber ${ }^{7}$ placed after the target. The charge collected on one system of plates in this chamber is measured with a current integrator. The sensitivity of this monitor was measured several times during each run by comparing it to a carefully calibrated ion chamber using a proton beam of reduced intensity $\left(10^{8} \mathrm{p} / \mathrm{sec}\right)$. The sensitivity never varied by more than $2 \%$ during a run. The linearity of the secondary emission chamber was checked over the range $2 \times 10^{9} \mathrm{p} / \mathrm{sec}$ to $10^{11} \mathrm{p} / \mathrm{sec}$ against the carbon activity produced in polythene plates. No deviation from linearity was found within the $2 \%$ precision of the measurements. The ion chamber in

TABLE I. Parameters used in the present experiment (see Fig. 1).

\begin{tabular}{|c|c|c|c|}
\hline & Run 1 & Run 2 & Run 3 \\
\hline $\begin{array}{l}\text { Target thickness } \\
\text { Collimator }(K 2) \text { opening } \\
\text { Thickness of tungsten jaws in } K 2 \\
\text { Protons } 100 \text { monitor counts } \\
\text { Counter } 1\left(\mathrm{H}^{3}\right) \\
\quad\left(\mathrm{He}^{3}\right) \\
\text { Counter } 2\left(\mathrm{H}^{3} \text { and } \mathrm{He}^{3}\right) \\
\text { Counter } 3\left(\mathrm{H}^{3}\right) \\
\quad\left(\mathrm{He}^{3}\right) \\
\text { Distance between counters } 1 \text { and } 2 \\
\text { Pulse-height system connected to counter } \mathrm{No} .\left(\mathrm{H}^{3}\right) \\
\text { Bending angle of the analyzing magnet }\end{array}$ & $\begin{array}{l}\quad 2.5 \mathrm{~cm} \\
2 \times 3 \text { and } 3 \times 2 \mathrm{~cm}^{2} \\
4 \text { and } 2 \mathrm{~cm} \\
8.6 \times 10^{12} \\
12 \times 12 \times 0.6 \mathrm{~cm}^{3} \\
12 \times 12 \times 0.2 \mathrm{~cm}^{3} \\
12 \times 18 \times 0.6 \mathrm{~cm}^{3} \\
13 \times 19 \times 0.6 \mathrm{~cm}^{3} \\
13 \times 19 \times 0.6 \mathrm{~cm}^{3} \\
390 \mathrm{~cm} \\
1 \\
2 \\
26.6^{\circ}\end{array}$ & $\begin{array}{l}0.8 \mathrm{~cm}^{2} \\
2 \times 3 \mathrm{~cm}^{2} \\
2 \mathrm{~cm} \\
5.6 \times 10^{12} \\
12 \times 12 \times 0.6 \\
12 \times 12 \times 0.2 \\
15 \times 20 \times 0.8 \\
15 \times 20 \times 0.8 \\
15 \times 20 \times 0.8 \\
390 \mathrm{~cm} \\
1 \\
2 \\
26.6^{\circ}\end{array}$ & $\begin{array}{l}0.8 \mathrm{~cm} \\
4 \times 3 \mathrm{~cm}^{2} \\
2 \mathrm{~cm} \\
5.6 \times 10^{12} \\
12 \times 12 \times 0.2 \\
12 \times 12 \times 0.2 \\
15 \times 20 \times 0.8 \\
15 \times 20 \times 0.8 \\
\cdots \\
250 \text { or } 150 \mathrm{~cm} \\
1 \text { (or } 2 \text { ) } \\
1 \text {. } \\
26.6^{\circ}\end{array}$ \\
\hline
\end{tabular}

${ }^{7}$ G. W. Tautfest and H. R. Fechter, Rev. Sci. Instr. 26, 229 (1955). 
TABLE II. Some properties of the $\mathrm{H}^{3}$ and $\mathrm{He}^{3}$ nuclei observed at $\theta_{\text {lab }}=14^{\circ}$. The range and energy loss are for plastic scintillator.

\begin{tabular}{lcccc}
\hline & \multicolumn{3}{c}{ Forward groups } & \multicolumn{2}{c}{ Backward groups } \\
& $\mathrm{H}^{3}$ & $\mathrm{He}^{3}$ & \multicolumn{1}{c}{$\mathrm{H}^{3}$} & \multicolumn{1}{c}{$\mathrm{He}^{3}$} \\
\hline$T(\mathrm{Mev})$ & 270 & 271 & 117 & 116 \\
$p c / Z(\mathrm{Mev})$ & 1250 & 625 & 819 & 407 \\
Time-of-flight/meter & 8.1 & 8.1 & 11.9 & 11.9 \\
$\quad(\mathrm{nsec})$ & 19.2 & 4.8 & 4.2 & 1.0 \\
Range $\left(\mathrm{g} \mathrm{cm}^{-2}\right)$ & 7.7 & 31 & 15.2 & 61 \\
Energy loss & & & & \\
$\quad\left(\mathrm{Mev} \mathrm{cm}^{2} \mathrm{~g}^{-1}\right)$ & & & & \\
\hline \hline
\end{tabular}

its turn was calibrated with a beam of several hundred protons/sec by counting the number of protons with a scintillation telescope. Repeated checks with a $\mathrm{Co}^{60}$ source showed a drift $<1 \%$ in the sensitivity of the ion chamber over a period of one year.

The maximum intensity of the beam is $10^{11} \mathrm{p} / \mathrm{sec}$. However, in general a lower flux had to be used to reduce accidentals and counting losses. The cyclotron produces 55 beam pulses a second with an average duration of about $50 \mu \mathrm{sec}$. This corresponds to a duty cycle of $1 / 400$ for the external proton beam.

After passing the monitor the beam continues in evacuated pipes until it leaves the experimental room. It is finally caught in a cave of concrete blocks 10 meters beyond this room.

\section{(C) Liquid Deuterium Target}

The liquid deuterium target was constructed according to a design of Whalin and Reitz. ${ }^{8}$ Deuterium gas is liquified by passing it through a tube which is kept at liquid hydrogen temperature. The liquid is collected in a small reservoir and then descends into a cylindrical appendix, which is used as the target, with the proton beam incident along its horizontal axis. The appendix can easily be emptied by closing a remotely controlled valve in its gas exhaust line. Filling and emptying of the target takes 30 seconds. A frequent alternation of target full and empty runs is thus possible.

In run 1 , the appendix was made of a copper ring 2 $\mathrm{cm}$ thick holding two Mylar foils $0.1 \mathrm{~mm}$ thick and 9 $\mathrm{cm}$ in diameter. With such an arrangement it is difficult to estimate the thickness of the deuterium layer through which the beam passes, as the windows bulge outwards under the influence of the pressure in the deuterium system, which is one-third of an atmosphere.

In runs 2 and 3 this difficulty was overcome by adding to the appendix two more Mylar windows, thus obtaining three separate compartments, and connecting the two outer compartments to the deuterium system in the gas phase (see Fig. 2). With this arrangement there is no pressure difference over the inside windows, which hold the liquid. The layer of liquid in this appendix is $0.80 \mathrm{~cm}$ thick and the diameter of the window is $10.5 \mathrm{~cm}$.

\footnotetext{
${ }^{8}$ E. A. Whalin and R. A. Reitz, Rev. Sci. Instr. 26, 59 (1955).
}
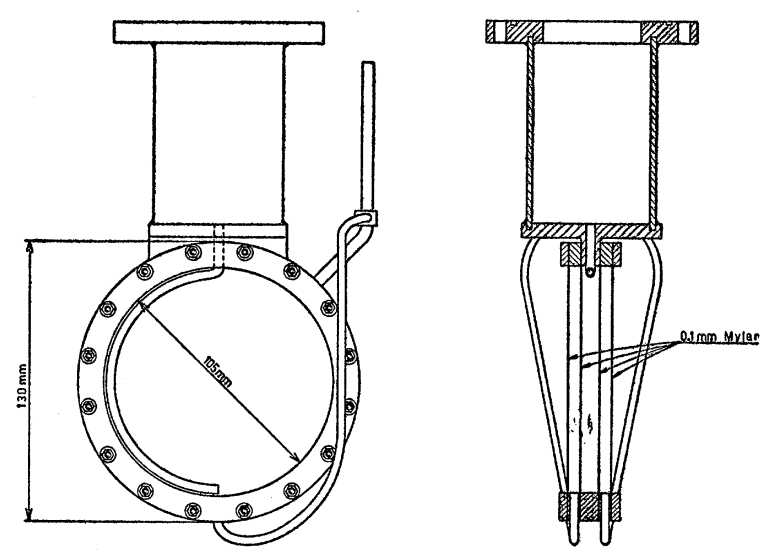

Fig. 2. The target appendix as used in runs 2 and 3.

\section{(D) Collimator}

The collimator which constitutes the entrance to the mass separator was placed 5 meters from the target. The dimensions of the collimator, which varied in the different runs, are given in Table I. The dimensions given in the following text serve only as an example. A 32-cm long copper collimator with an opening $4 \times 5 \mathrm{~cm}^{2}$ was provided with tungsten jaws leaving a $3 \times 4 \mathrm{~cm}^{2}$ gap in order to reduce the seattering from the inner faces. Four-cm thick jaws are sufficient to stop both the $\mathrm{He}^{3}$ and $\mathrm{H}^{3}$ particles; two-cm jaws are thick enough to stop the $\mathrm{He}^{3}$ particles and to degrade the energy of the $\mathrm{H}^{3}$ particles sufficiently for the magnet to bend them away from the counter telescope. The edge effects in the collimator, that is the fraction of particles that hit the tungsten jaws and are still counted, can be calculated to lead to an uncertainty in the effective solid angle of $1 \%$ for the $\mathrm{H}^{3}$ particles. For $\mathrm{He}^{3}$ particles these effects are negligible.

\section{(E) Analyzing Magnet}

The path of the particles through the magnet was studied with wire measurements before each run. The edges of the wedge-shaped pole faces were set at such an angle to the beam, that maximum double-focusing was obtained. In the horizontal plane it proved possible to produce an image of the target at the position of counter 2 . The focusing in the vertical plane, which was less effective, was also checked by setting the magnet current for elastically scattered protons and measuring the height of the beam spot by moving counters 2 and 3 up and down. The results from these vertical position curves, obtained with both a $3 \times 4 \mathrm{~cm}^{2}$ and a $4 \times 1 \mathrm{~cm}^{2}$ collimator, agreed well with the predictions of the wire measurements. The dimensions of counters 2 and 3 were based on the wire measurements, taking into account the energy spread of the particles leaving the target and the multiple scattering produced in the beam path. 


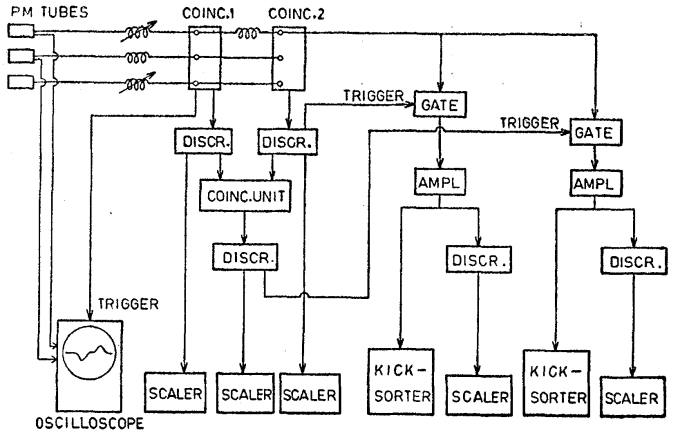

FIG. 3. Block diagram of electronics.

\section{(F) Counters and Electronics}

The counters were plastic scintillators connected by Lucite light guides to RCA-6810 A photomultipliers. The dimensions of the counters used in the different runs are listed in Table $\mathrm{I}$.

To minimize multiple scattering, counter 1 was kept as thin as the requirement of sufficient light for pulseheight analysis permitted. The absorption of $\mathrm{He}^{3}$ particles in plastic scintillator was measured by placing a $8-\mathrm{mm}$ thick plate between counters 2 and 3 . The reduction in the number of triple coincidences was only $(0.4 \pm 1.2) \%$. From the slope of a range curve on tritons, it follows that absorption of tritons can also be neglected.

A block diagram of the electronics is shown in Fig. 3. To achieve complete separation of mass three particles from protons and deuterons by time of flight, a coincidence circuit with a resolving time of a few nanoseconds (nsec) is needed. The coincidence circuit developed by Wenzel $^{9}$ meets this requirement. The circuits were tested by connecting them to a three-counter telescope in the direct proton beam of reduced intensity. Figure 4 shows the ratio of the numbers of triple and double coincidences as a function of delay inserted in the cables to the third counter. A $100 \%$ counting efficiency was obtained over a flat top of $2 \mathrm{nsec}$ and the width at half height is $5.5 \mathrm{nsec}$. A further reduction of the width could only be obtained by sacrificing stability. When a resolving time of $4 \mathrm{nsec}$ was desired, two coincidence units were used, one with an extra delay of $1.5 \mathrm{nsec}$ inserted in the connection to one counter and slow coincidences between the two circuits were counted.

A drawback of this type of coincidence circuit is that also single pulses cause a dead time. As the clipping is done in the anode lines of the $E 180 F$ limiting tubes, the input channel is blocked for the duration of the unclipped pulse.

The high voltages on the photomultipliers were set such that the particles to be counted produced 4 -volt pulses at the input of the coincidence circuit. This

\footnotetext{
${ }^{9}$ W. A. Wenzel, University of California Radiation Laboratory Report UCRL-8000, 1957 (unpublished).
}

procedure minimizes the electronic bias in passing from $\mathrm{H}^{3}$ to $\mathrm{He}^{3}$.

Pulses are selected for pulse-height analysis by 60 nsec wide gates of the pulse transformer type. ${ }^{10}$ When triggered by an output of the coincidence circuit these gates pass the pulses of one of the counters both to an integral discriminator and to a multichannel pulseheight analyzer.

Pulses from the last dynodes of two counters are displayed with properly chosen delays on an Edgerton Type 2236 travelling-wave oscilloscope triggered by another output of a coincidence circuit. Variations in flight time of 1 nsec may be measured by scanning the films on which these traces are recorded.

\section{MEASUREMENTS}

\section{(A) General}

Each measurement of the differential cross sections of the two reactions was preceded by a series of checks which established the correct measuring conditions. The length of cable to counter 1 was varied to find the exact time-of-flight, the field in the analyzing magnet was varied to bend the beams over exactly $26.6^{\circ}$, and the counters 2 and 3 were moved both vertically and horizontally to make the center of the beam spot coincide with the center of the counters. All of these measurements in which the counting rate is measured as a function of a certain parameter also serve a second purpose: to ascertain that all the $\mathrm{H}^{3}$ (or $\mathrm{He}^{3}$ ) particles passing the collimator are counted, and that all counts correspond to $\mathrm{H}^{3}$ (or $\mathrm{He}^{3}$ ) particles. Ideally one hopes to find a clear separation between groups of different

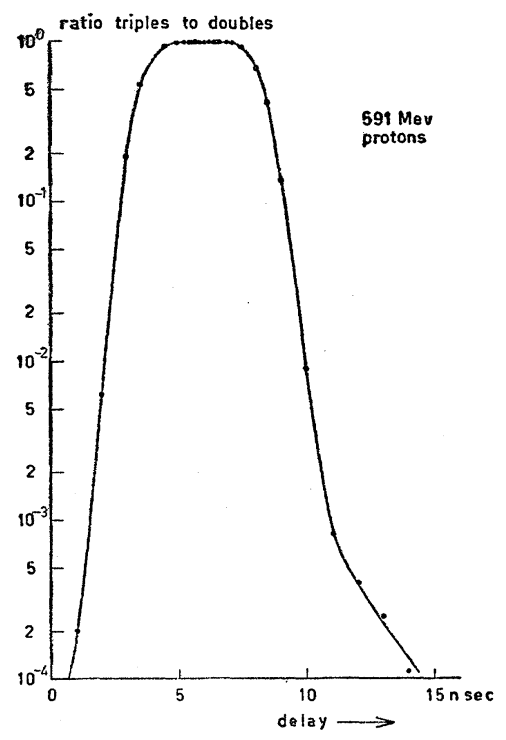

FIG. 4. Delay curve of the coincidence circuit measured with 591-Mev protons.

${ }^{10}$ F. P. G. Valckx and A. Dymanus, Nuclear Instr. 7, 197 $(1960)$. 


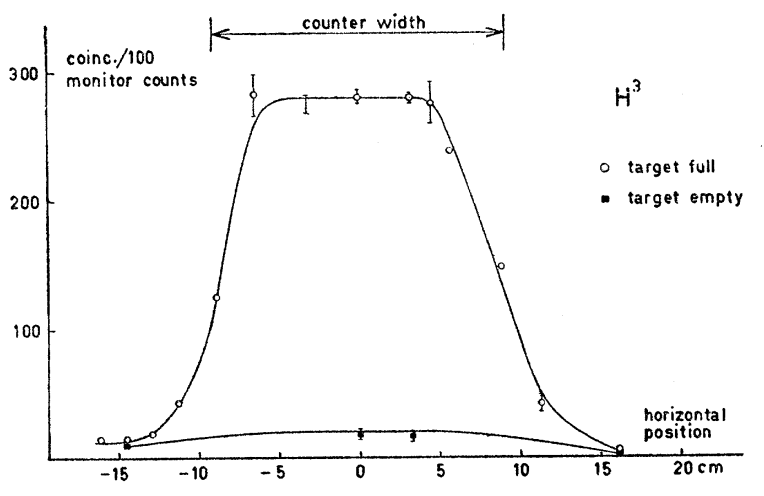

(a)

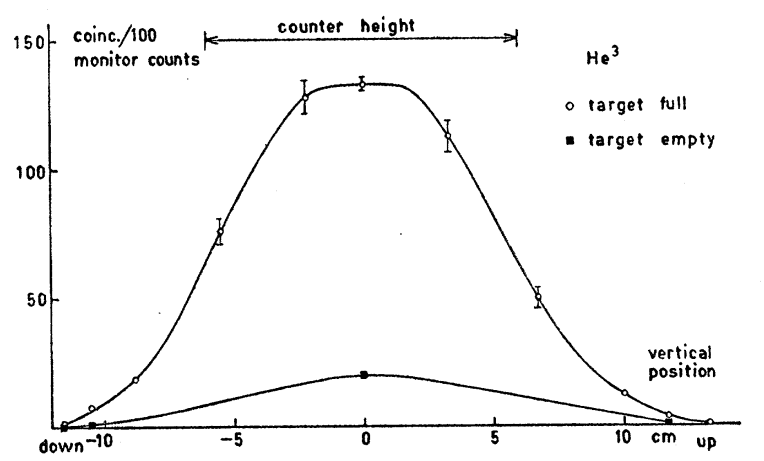

(b)

particles in the delay and magnet curves, and the "marginal" counts with target full and target empty should cancel. Marginal counts are the counts obtained when counters 2 and 3 are displaced horizontally or vertically by their own width or height from the central position.

Every measurement consisted of seven readings: the counts from the two coincidence circuits, from the mixed coincidence circuit, from the two integral discriminators and from the two pulse-height analyzers. The readings of the last four were always equal to within $1 \%$ for measurements in the central positions after correction for counting losses in the kicksorters. The proper functioning of the electronics was checked continuously in this way.

The pulse-height measurement supplies independent information on the identification of the $\mathrm{He}^{3}$ particles, as only double-charged particles of the right velocity give the correct pulse height. Unfortunately, the pulseheight analysis does not distinguish tritons from protons and deuterons of the same velocity. Even for the $\mathrm{H}^{3}$ settings the pulse-height spectra help to detect the presence of counts caused by accidental coincidences or by scattered protons and deuterons with a velocity different from the $\mathrm{H}^{3}$ velocity, but still compatible with the resolving time of the coincidence circuit.

The measurements were always made with the same counter connected to the pulse-height system, but during the initial checks each counter was connected

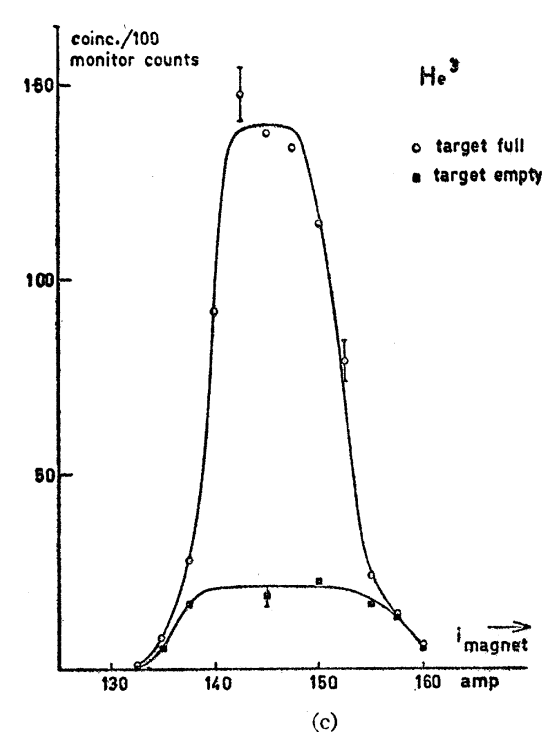

FIG. 5. Number of triple coincidences per 100 monitor counts as a function of (a) horizontal displacement of counters 2 and 3 for $\mathrm{H}^{3}$ settings, (b) vertical displacement of counters 2 and 3 for $\mathrm{He}^{3}$ settings and (c) analyzing magnet current for $\mathrm{He}^{3}$.

in turn to the pulse-height system to verify that the $\mathrm{H}^{3}$ and $\mathrm{He}^{3}$ peaks came in the right channels and were well separated from other groups.

\section{(B) First Run}

A short report ${ }^{6}$ on the results obtained in this run has been published before, but some more detailed information can be given here. Figures 5(a), (b), and (c) showing a horizontal position, a vertical position and a magnet curve, indicate to what extent the ideal situation of clean, uncontaminated $\mathrm{H}^{3}$ and $\mathrm{He}^{3}$ detection has been approached. The pulse-height spectra are presented in Figs. 6(a) and (b).

A check on the flatness of the delay curve and thus the $100 \%$ efficiency of the coincidence circuit was made by making a measurement with the delay shifted $0.7 \mathrm{nsec}$ from the central position. Also two different collimators were used to demonstrate the absence of "collimator effects." The results obtained in these three series of measurements are given in Table III. As no systematic deviations are observed, the data of the three series have been averaged to give the result $(263.7 \pm 2.8) \mathrm{H}^{3}$ and $(115.0 \pm 2.0) \mathrm{He}^{3}$ particles per 100 monitor counts and thus $R_{\mathrm{lab}}=2.29 \pm 0.05$. To the statistical error a possible systematic error has to be added, which comes mainly from the uncertainty about the nature of the marginal counts (see reference 6). The total standard error thus becomes 5\%. After 


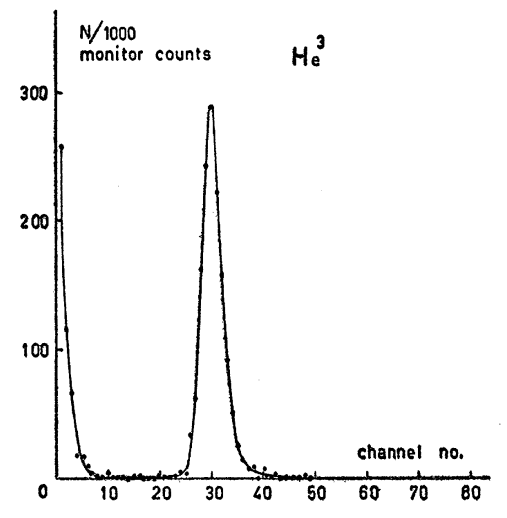

(a)

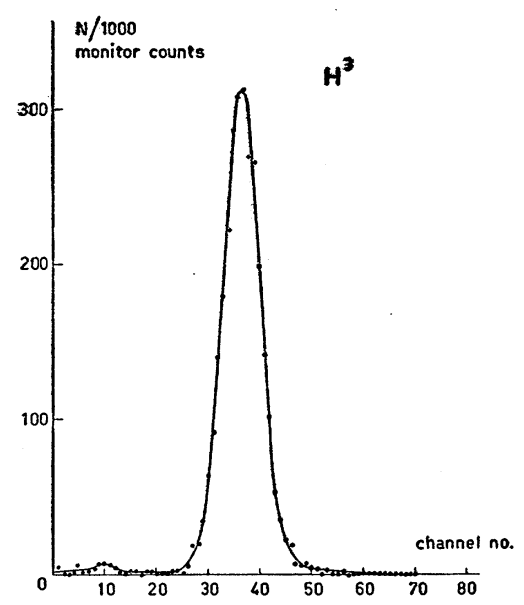

FIG. 6. Pulse-height spectrum in counter 2, gated by triple coincidences, for (a) $\mathrm{H}^{3}$ settings, (b) $\mathrm{He}^{3}$ settings. The number of counts per channel is given for 1000 monitor counts.

transformation to the c.m. system the final result of this run for the ratio of the differential cross sections of reactions (1) and (2) at $\theta_{\mathrm{c} . \mathrm{m} .}=52^{\circ}$ is : $R=2.26 \pm 0.11$.

\section{(C) Second Run}

The aims of this run were twofold: to measure the variation of the cross section of reaction (1) with energy and to repeat the measurement of $R$ at a slightly different angle $\left(\theta_{\mathrm{lab}}=14^{\circ}\right)$ to check possible systematic errors. As during this run pictures were taken of all pulses on the travelling wave oscilloscope, it was considered unnecessary to reduce the beam intensity

TABLE III. Results of three series of measurements in run 1 (see text). The number of counts is given for 100 counts of the beam monitor $\left(8.6 \times 10^{12}\right.$ protons $)$.

\begin{tabular}{crrr}
\hline \hline & \multicolumn{1}{c}{$A$} & \multicolumn{1}{c}{$B$} & \multicolumn{1}{c}{$C$} \\
\hline $\mathrm{H}^{3}$ target full & $281.6 \pm 3.8$ & $280.1 \pm 3.8$ & $271.8 \pm 7.4$ \\
target empty & $17.6 \pm 1.9$ & $13.7 \pm 1.5$ & $19.5 \pm 3.2$ \\
$\mathrm{He}^{3}$ target full & $137.8 \pm 2.4$ & $134.4 \pm 5.2$ & $134.9 \pm 3.2$ \\
target empty & $22.6 \pm 1.8$ & & $19.9 \pm 1.6$ \\
\hline
\end{tabular}

until the number of accidentals became negligibly small, since a correction could be made for the number of accidentals on the basis of film scanning.

The production of a $581-\mathrm{Mev}$ proton beam geometrically similar to the $591-\mathrm{Mev}$ beam proved to be time consuming. As a consequence there was no time left to alternate measurements on $\mathrm{H}^{3}$ and $\mathrm{He}^{3}$ as had been done in the first run, and only four series of measurements were made:
(A) $p+d \rightarrow \mathrm{H}^{3}+\pi^{+}$
$E_{p}=591 \mathrm{Mev}$
(B) $p+d \rightarrow \mathrm{He}+\pi^{0}$
$E_{p}=591 \mathrm{Mev}$;
(C) $p+d \rightarrow \mathrm{H}^{3}+\pi^{+}, \quad E_{p}=581 \mathrm{Mev}$;
(D) $p+d \rightarrow \mathrm{H}^{3}+\pi^{+}, \quad E_{p}=591 \mathrm{Mev}$ [repeat of (A)].

Runs $A, C$, and $D$ all contained $5 \%$ accidentals with target full and $1 \%$ with target empty, run $2 B$ had $1 \%$ accidentals both with target full and empty. Table IV gives the corrected results and shows a discrepancy between the measurements $A$ and $D$ which is nearly twice the standard error. This discrepancy is attributed to a decrease in the effective thickness of the target, due to an observed increase in bubbling in the liquid deuterium. This bubbling could have rather a large influence because the target used in this run was only 8 $\mathrm{mm}$ thick. As there were equal time intervals between measurements $A, B, C$, and $D$, it seems preferable to calculate the ratio from a comparison of $B$ with $\left(\frac{2}{3} A+\frac{1}{3} D\right)$. Thus one obtains $R_{\text {lab }}=(57.8 \pm 2.5) /(27.0$ $\pm 1.0)=2.14 \pm 0.12$.

Just as in the first run, measurements were made with the counters displaced by their own width in four directions. A study of the corresponding oscilloscope traces shows that most of the marginal counts can be discarded as due to accidentals or particles other than $\mathrm{H}^{3}$ or $\mathrm{He}^{3}$. Actually no $\mathrm{H}^{3}$ or $\mathrm{He}^{3}$ nuclei at all were found above and below counter 2, whereas in the horizontal marginal positions a few percent of what is found in the central position seem to remain both with target full and empty. Again it is preferred not to apply small corrections, which would cancel in the ratio, but rather to account for these dubious counts by assigning an extra error of $2 \%$ to $R$. The accidentals mentioned above prevent the delay curve from going to zero between the triton and deuteron group. The uncertainty in the number of accidentals to be subtracted is estimated to be $1 \%$ of the total counting rate. Finally an uncertainty of $3 \%$ is attributed to the interpolation procedure which was followed to account for the increased bubbling in the target. The standard error in $R$ is thus raised to $7 \%$, and the result after transformation to the center-ofmass system becomes $R=2.10 \pm 0.14$ for $\theta_{\text {c.m. }}=67^{\circ}$, with a proton energy of $591 \mathrm{Mev}$ for both processes.

The energy dependence is calculated from a comparison of run $C$ with $\left(\frac{1}{3} A+\frac{2}{3} D\right)$. A $10-\mathrm{Mev}$ decrease in $E_{p}$ results in an increase in the cross section of reaction (1) of $(4.9 \pm 2.2) / 55.8=(8.8 \pm 4.0) \%$. The experimental 
TABLE IV. Results of the measurements in run 2. The number of counts is given for 100 counts of the beam monitor $\left(5.6 \times 10^{12}\right.$ protons).

\begin{tabular}{lccc}
\hline \hline & Full & Empty & Full-empty \\
\hline (A) $E_{p}=591 \mathrm{Mev}, \mathrm{H}^{3}$ & $69.9 \pm 2.6$ & $10.2 \pm 1.2$ & $59.7 \pm 2.9$ \\
$(B) E_{p}=591 \mathrm{Mev}, \mathrm{He}^{3}$ & $43.9 \pm 0.7$ & $16.9 \pm 0.7$ & $27.0 \pm 1.0$ \\
$(C) E_{p}=581 \mathrm{Mev}, \mathrm{H}^{3}$ & $74.6 \pm 1.1$ & $13.9 \pm 0.9$ & $60.7 \pm 1.4$ \\
$(D) E_{p}=591 \mathrm{Mev}, \mathrm{H}^{3}$ & $65.0 \pm 1.2$ & $11.1 \pm 1.0$ & $53.9 \pm 1.6$ \\
\hline
\end{tabular}

conditions in the measurement at $591 \mathrm{Mev}$ and 581 Mev are identical, so that all systematic errors should cancel. The c.m. solid angle is, however, increased by $2.3 \%$ when the proton energy is reduced by $10 \mathrm{Mev}$ and thus the differential cross section in the c.m. system is only increased by $(6.3 \pm 4.0) \%$ for the $10-\mathrm{Mev}$ change in proton energy.

\section{(D) Third Run}

This time the backward groups of $\mathrm{H}^{3}$ and $\mathrm{He}^{3}$ were investigated at $14^{\circ}$ lab angle corresponding to $143^{\circ} \mathrm{c} . \mathrm{m}$. angle. The experiment has to be adapted to the much lower energy of these groups. For instance, the thin 8-mm target has to be used to keep the energy spread in the $\mathrm{He}^{3}$ beam within reasonable limits. Improvements in the radiation shield reduced the bubbling in the liquid deuterium compared to the second run. The larger energy loss per $\mathrm{cm}$ produces bigger light pulses in the scintillators, permitting the use of lower voltages on the photomultiplier tubes. The relative rate of single pulses above a certain voltage is correspondingly reduced and thus the number of accidental counts was always negligible during this run. This is especially helpful for the $\mathrm{He}^{3}$ particles, since their range is so short that only a two-counter telescope can be used.

Due to the lower velocities of the particles in the backward groups complete time-of-flight separation can be achieved with the distance between counters 1 and 2 reduced to 2.5 meters. This reduction in distance compensates for the increase in multiple scattering in the first counter due to the lower energy of the particles. Successful measurements were made under these conditions for $\mathrm{H}^{3}$. For $\mathrm{He}^{3}$, however, the spreading out of the beam was somewhat worse than expected, about $10 \%$ of the particles being lost above and below counter 2 . The distance was thereupon further decreased to 1.5 meters, which reduced the loss of $\mathrm{He}^{3}$ particles to $2 \%$. The pulse-height criterium makes an unambiguous identification of the $\mathrm{He}^{3}$ particles still possible.

A measurement was also made on tritium with $1.5-\mathrm{m}$ separation between the first two counters. The films with oscilloscope traces make it possible to correct for the incomplete electronic time-separation between tritons and deuterons. These corrections amount to $4.6 \%$ for target full and $9.8 \%$ for target empty measurements, whereas the film scanning revealed corrections of only $0.8 \%$ for target full and $1.4 \%$ for target empty
TABLE V. Results of the measurements in run 3. The number of counts is given for 100 counts of the beam monitor $\left(5.6 \times 10^{12}\right.$ protons).

\begin{tabular}{|c|c|c|c|c|c|}
\hline \multicolumn{3}{|r|}{$\begin{array}{l}\text { Distance between } \\
\text { counters } 1 \text { and } 2\end{array}$} & Full & Empty & Full-empty \\
\hline $\begin{array}{l}\text { (A) } \\
\text { (B) } \\
\text { (D) }\end{array}$ & $\begin{array}{l}\mathrm{H}^{3} \\
\mathrm{He}^{3} \\
\mathrm{He}^{3} \\
\mathrm{H}^{3} \\
\mathrm{H}^{3} \mathrm{a} \\
\mathrm{He}^{3}\end{array}$ & $\begin{array}{l}250 \mathrm{~cm} \\
250 \mathrm{~cm} \\
150 \mathrm{~cm} \\
150 \mathrm{~cm} \\
\text { verage of } A \text { and } D \\
\text { average of } B \text { and } C\end{array}$ & $\begin{array}{l}406.3 \pm 3.4 \\
231.6 \pm 9.2 \\
263.9 \pm 2.4 \\
426.1 \pm 6.7\end{array}$ & $\begin{array}{r}98.6 \pm 2.6 \\
77.0 \pm 8.8 \\
114.2 \pm 1.9 \\
114.7 \pm 5.0\end{array}$ & $\begin{array}{l}307.7 \pm 4.3 \\
154.6 \pm 13 \\
149.7 \pm 3.1 \\
311.4 \pm 8.4 \\
308.4 \pm 3.8 \\
150.0 \pm 3.0\end{array}$ \\
\hline
\end{tabular}

for the tritium measurements with $2.5-\mathrm{m}$ flight path. The results obtained after these corrections, given in Table $\mathrm{V}$, lead to $R_{\mathrm{lab}}=2.06 \pm 0.05$ and $R_{\mathrm{c} . \mathrm{m} .}=1.99$ \pm 0.05 .

In the search for potential sources of systematic errors the tritium measurements have been made partly with counter 1 and partly with counter 2 connected to the pulse-height system. The results 404.4 \pm 4.5 and $408.9 \pm 5.1$ agree well. The uncertainty as to whether all the $\mathrm{H}^{3}$ and $\mathrm{He}^{3}$ particles and only those particles have been detected, is expressed in a $2 \%$ error. Another uncertainty of $2 \%$ stems from a discrepancy between two calibrations of the beam monitor. From direct observation there was no indication of a change in bubbling rate in the liquid deuterium during this run. In view of the difficulties in run 2 , however, an error of $2 \%$, the maximum uncertainty that the consistency of the measurements permits, was assigned to $R$ for variations in the target thickness.

In the two previous runs the tacit assumption has been made that the differential cross sections of these reactions vary little with angle in the region of interest. The $0.3^{\circ}$ difference in the c.m. angle for $\mathrm{He}^{3}$ and $\mathrm{H}^{3}$ could hence be neglected. The equality of the cross sections at $52^{\circ}$ and $67^{\circ}$ found in this experiment (see Table VI) and the measurements by Frank et al. ${ }^{2}$ at $340 \mathrm{Mev}$ support this assumption. The backward $\mathrm{H}^{3}$ and $\mathrm{He}^{3}$ groups correspond to the forward pion groups and, for these angles, a strong dependence of the cross sections on angle has been observed., ${ }^{2,11}$ From the data at $450 \mathrm{Mev}$ and $660 \mathrm{Mev}$ the change in cross section between the $\mathrm{H}^{3}$ angle $\left(142.7^{\circ}\right)$ and $\mathrm{He}^{3}$ angle $\left(143.2^{\circ}\right)$ can be estimated as $(3.0 \pm 2.0) \%$ with sufficient reliability.

The standard error in this run is thus $4.5 \%$ and the ratio of the cross sections of the two reactions for $\theta_{\text {c.m. }}=143^{\circ}$ becomes $R=2.05 \pm 0.09$.

\section{(E) Accidentals}

High single rates in the counters cause accidental coincidences and, due to blocking of the single inputs of the coincidence circuits, also counting losses. This problem is most serious for the $\mathrm{H}^{3}$ groups in the forward direction when high voltages on the multipliers are used.

${ }^{11} \mathrm{Iu} . \mathrm{K}$. Akimov, O. V. Savchenko, and L. M. Soroko, Joint Institute for Nuclear: Researçh, Dubna, $P-413,1959$ (unpublished). 

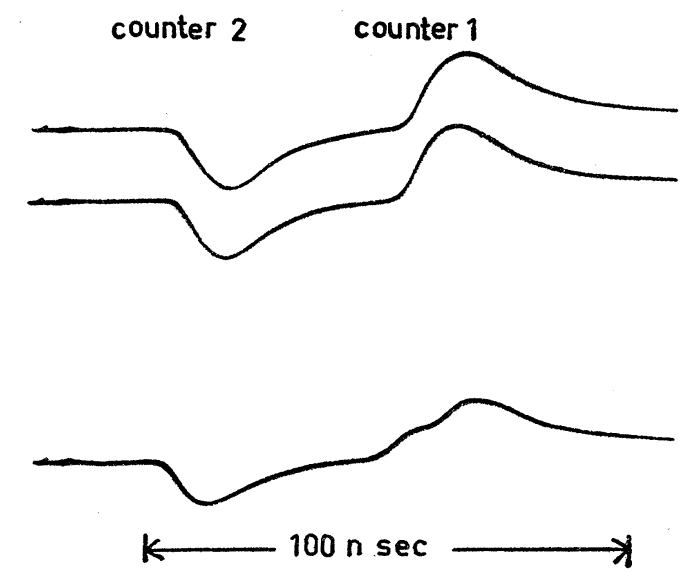

FIG. 7. Traces from oscilloscope film showing the pulses from a triton in counters 1 and 2 . The third trace was triggered by an accidental coincidence as described in the text.

In the present telescope accidental triple coincidences are mainly produced by chance coincidences of a single in counter 1 with a true double in counters 2 and 3 . In general, the beam intensity was reduced until less than $1 \%$ accidentals could be expected from the measured singles and doubles rates. Accidentals will betray themselves by having the wrong pulse height and by preventing the delay curve from dropping to zero on its sides. Both facts were indeed observed during run 1, when the beam intensity was $8 \times 10^{10}$ protons $/ \mathrm{sec}$. A beam of only $10^{10}$ protons/sec had to be used for the $\mathrm{H}^{3}$ measurement in run 1 . In runs 2 and 3 accidentals are also easily recognized in the film scanning. They show up by having wrong pulse heights in both counters and wrong timing. The complicated time structure of the beams of particles through the counters gives rise in run 2 to accidentals which are mostly of one special type (Fig. 7). A proton and a deuteron produced in the same rf cycle of the cyclotron pass through the first counter. The deuteron has the momentum selected by the magnet and passes also counters 2 and 3 . The time difference between the proton crossing counter 1 and the deuteron crossing counters 2 and 3 corresponds to the selected time-of-flight. Thus the coincidence circuit fires and opens the 60 nsec wide gate, which passes both pulses from counter 1 . The slow pulse-height system sees these two pulses as one pulse of $\mathrm{H}^{3}$ pulse height. The danger is that it is easy to obtain misleading results, since accidental coincidences of this kind disappear when one inserts an extra $60 \mathrm{nsec}$ (the interval between two rf bursts) in the delay cable.

The effect of accidentals is partly counteracted by counting losses, but in general the situation prevailed in which there were more accidentals than losses.

\section{(F) Absolute Cross Sections}

As the sensitivity of the secondary emission chamber during each run was compared to an absolutely cal- ibrated ionization chamber, the absolute values of the differential cross sections can be calculated. The pertinent data are given in Table VI. The errors in the cross sections have been obtained by combining the statistical error with a $3 \%$ uncertainty in the calibration of the monitor, a $2 \%$ error to account for errors in the identification of $\mathrm{H}^{3}$ and $\mathrm{He}^{3}$ particles, and a $10 \%$ uncertainty in the effective thickness of the target in runs 1 and 2 and a $3 \%$ uncertainty in run 3 .

\section{DISCUSSION AND CONCLUSIONS}

The precision obtained in the measurements of the ratio $R=d \sigma\left(p+d \rightarrow \mathrm{H}^{3}+\pi^{+}\right) / d \sigma\left(p+d \rightarrow \mathrm{He}^{3}+\pi^{0}\right)$ is of the same order of magnitude as the Coulomb and mass corrections. A strict test of the hypothesis of charge independence thus requires a precise knowledge of these effects. According to calculations by Köhler, ${ }^{12}$ of the CERN Theoretical Division, only four effects cause non-negligible corrections to $R$. The main correction (a) stems from the difference in triton and helium wave functions. A further correction (b) is based on the plausible but not indisputable assumption that the two reactions should be compared for the same momentum of the outgoing pions, thus reaction (1) at $E_{p}=591$ $\mathrm{Mev}$ with reaction (2) at $E_{p}=587 \mathrm{Mev}$. From the measured $(6.3 \pm 4.0) \%$ increase in the cross section for a $10-\mathrm{Mev}$ decrease in $E_{p}$, a correction of $3.6 \%$ is computed. The difference in the phase space factors in the two reactions causes another correction (c) of $-0.7 \%$. The last correction (d), which accounts for the fact that the mesons come out at slightly different c.m. angles in the two reactions, is only of importance for the backward groups measured in the third run. The available experimental data permit, however, a more direct estimate of this correction. It is evident that both in the calculation of these corrections and in the neglect of other effects, use has been made of various assumptions and models. The uncertainty in the validity of these assumptions is expressed in an estimated error of $3 \%$ in the results.

In the comparison of the experimental and theoretical values of the ratio that follows, correction (d) has already been applied to $R_{\exp }$, and $R_{\text {theor }}$ thus includes only Köhlers corrections (a), (b) and (c).

\begin{tabular}{ccc}
$\theta_{\text {c.m. }}\left(\mathrm{H}^{3}, \mathrm{He}^{3}\right)$ & $R_{\exp }$ & $R_{\text {theor }}$ \\
\hline $52^{\circ}$ & $2.26 \pm 0.11$ & $2.21 \pm 0.07$ \\
$67^{\circ}$ & $2.10 \pm 0.14$ & $2.22 \pm 0.07$ \\
$143^{\circ}$ & $2.05 \pm 0.09$ & $2.18 \pm 0.07$
\end{tabular}

As the variation with angle of $R_{\text {theor }}$ is much smaller than the uncertainty in $R_{\text {theor }}$, one may also compare the values averaged over the three angles of observation:

$$
R_{\text {exp }}=2.13 \pm 0.06 \text { with } R_{\text {theor }}=2.20 \pm 0.07 \text {. }
$$

${ }^{12}$ H. S. Köhler, Phys. Rev. 118, 1345 (1960). 
Table VI. Absolute differential cross sections of the reactions $p+d \rightarrow \mathrm{H}^{3}+\pi^{+}(1)$ and $p+d \rightarrow \mathrm{He}^{3}+\pi^{0}(2)$ in $\mu \mathrm{b} / \mathrm{sr}$. The errors in the cross sections include statistical and systematic errors, the errors in the numbers of counts are statistical only.

\begin{tabular}{lccc}
\hline \hline & Run 1 & Run 2 & Run 3 \\
\hline Lab angle $\theta$ & $11.3^{\circ}$ & $14.0^{\circ}$ & $14.0^{\circ}$ \\
C.m. angle $\theta_{1}^{*}\left(\mathrm{H}^{3}\right)$ & $51.7^{\circ}$ & $67.2^{\circ}$ & $142.7^{\circ}$ \\
C.m. angle $\theta_{2}{ }^{\circ}\left(\mathrm{He}^{3}\right)$ & $51.4^{\circ}$ & $66.7^{\circ}$ & $143.2^{\circ}$ \\
Solid angle in sr & $2.27 \times 10^{-5}$ & $2.32 \times 10^{-5}$ & $4.64 \times 10^{-5}$ \\
Number of protons/100 counts of the monitor & $8.6 \times 10^{12}$ & $5.6 \times 10^{12}$ & $4.0 \times 10^{12}$ \\
Number of $\mathrm{D}$ atoms $/ \mathrm{cm}^{2}$ & $12.7 \times 10^{22}$ & $4.0 \times 10^{22}$ & $308.4 \pm 3.8$ \\
Number of $\mathrm{H}^{3} / 100$ counts of the monitor & $263.7 \pm 2.8$ & $59.7 \pm 2.9$ & $29.7 \pm 1.5$ \\
{$\left[d \sigma_{1}(\theta) / d \Omega\right]_{\text {lab }}$} & $10.6 \pm 1.2^{\mathrm{a}}$ & $1.5 \pm 1.4$ & $2.92 \pm 0.14$ \\
{$\left[d \sigma_{1}\left(\theta_{1}^{*}\right) / d \Omega\right]_{\text {c.m. }}$} & $0.50 \pm 0.06$ & $0.49 \pm 0.06$ & $150.0 \pm 3.0$ \\
Number of $\mathrm{He} \mathrm{e}^{3} / 100$ counts of the monitor & $115.0 \pm 2.0$ & $27.0 \pm 1.0$ & $14.4 \pm 0.7$ \\
{$\left[d \sigma_{2}(\theta) / d \Omega\right]_{\text {lab }}$} & $4.6 \pm 0.5$ & $5.2 \pm 0.6$ & $1.45 \pm 0.07$ \\
{$\left[d \sigma_{2}\left(\theta_{2}{ }^{*}\right) / d \Omega\right]_{\text {c.m. }}$} & $0.22 \pm 0.02$ & $0.23 \pm 0.02$ & \\
\hline \hline
\end{tabular}

The value of $14 \mu \mathrm{b} / \mathrm{sr}$ given in our preliminary report (reference 6) is incorrect.

The present experiment thus confirms the validity of the hypothesis of charge independence with an experimental precision which is equal to the uncertainty in the theoretical prediction. Crewe $e t a l .{ }^{3}$ in a recent experiment measured the same ratio $R$ with a somewhat different technique at a proton energy of $450 \mathrm{Mev}$. Using momentum selection and pulse-height analysis to identify the $\mathrm{H}^{3}$ and $\mathrm{He}^{3}$ nuclei, and $\mathrm{CD}_{2}-\mathrm{C}$ as a deuterium target, they obtain $R=2.13 \pm 0.15$ at $\theta_{\text {c.m. }}=140^{\circ}$. Making the reasonable assumption that the Coulomb and mass corrections do not depend strongly on the energy of the incident protons, the good agreement between this result and that of the present experiment lends further support to the hypothesis of charge independence.

\section{ACKNOWLEDGMENTS}

Without a reliable estimate of the electromagnetic corrections to the ratio $R$, this experiment would have been inconclusive as a test of charge independence. The authors wish to express their gratitude to Dr. H. S. Köhler for performing these essential calculations and for numerous discussions. The authors also wish to thank Professor G. Bernardini for helpful discussions, Dr. G. Fidecaro, who kindly lent us his hydrogen target and Dr. B. Hedin for his work on the extraction of the proton beam.

The help of Dr. G. Culligan during the third run is gratefully acknowledged. The technical assistance of Mr. V. Beck, Mr. R. Sugden, Mr. P. Actis, and the co-operation of the cyclotron staff were substantial contributions to the success of the experiment. 